Supporting Information

\title{
Transport Evidence for Sulfur Vacancies as the Origin of Unintentional $n$-Type Doping in Pyrite $\mathrm{FeS}_{2}$
}

\author{
Bryan Voigt ${ }^{1}$, William Moore ${ }^{1}$, Michael Manno ${ }^{1}$, Jeff Walter ${ }^{1,2}$ \\ Jeff D. Jeremiason ${ }^{3}$, Eray S. Aydil* ${ }^{1,4}$, and Chris Leighton*1 \\ ${ }^{1}$ Department of Chemical Engineering and Materials Science, \\ University of Minnesota, Minneapolis, MN 55455, USA \\ ${ }^{2}$ Department of Physics and Astronomy, Carleton College, Northfield, MN 55057, USA \\ ${ }^{3}$ Department of Chemistry, Gustavus Adolphus College, Saint Peter, MN 56082, USA \\ ${ }^{4}$ Department of Chemical and Biomolecular Engineering, New York University Tandon School of \\ Engineering, Brooklyn, NY 11201, USA
}

*Corresponding authors: leighton@umn.edu, aydil@nyu.edu 


\section{A. Reduction to FeS2-8 vs. Decomposition to Pyrrhotite Fe1-xS}

This section provides calculated sulfur vacancy $\left(\mathrm{V}_{\mathrm{S}}\right)$ concentration profiles under different vacuum annealing conditions, comparing them to estimates of the $\mathrm{Fe}_{1-\mathrm{x}} \mathrm{S}$ layer thickness formed by decomposition of $\mathrm{FeS}_{2}$ to pyrrhotite. The time-dependent diffusion equation,

$$
\frac{\partial c}{\partial t}=D \frac{\partial^{2} c}{\partial z^{2}},
$$

was solved with boundary conditions

$$
c=c_{s}=5 \times 10^{22} \mathrm{~cm}^{-3} \text { at } z=0
$$

and,

$$
c=c_{\infty}=1 \times 10^{17} \mathrm{~cm}^{-3} \text { as } z \rightarrow \infty
$$

where $c$ is the $\mathrm{V}_{\mathrm{S}}$ concentration, $D$ is the $\mathrm{V}_{\mathrm{S}}$ diffusion coefficient, $t$ is time, and $z$ is the distance from the crystal surface into the crystal interior. The value $c_{s}=5 \times 10^{22} \mathrm{~cm}^{-3}$ is the maximum concentration of $\mathrm{V}_{\mathrm{S}}$ possible, corresponding to $\delta=2$ in $\mathrm{FeS}_{2-\delta} . c_{\infty}=1 \times 10^{17} \mathrm{~cm}^{-3}$ is the background $\mathrm{V}_{\mathrm{S}}$ concentration, set at this value due to observation of $300 \mathrm{~K}$ electron densities of $10^{16}-10^{18} \mathrm{~cm}^{-3}$ in this work. Note that, as seen clearly below, changing $c_{\infty}$ has little impact on the $\mathrm{V}_{\mathrm{S}}$ diffusion length or concentration profile, other than lifting the background concentration at high $z$. Diffusion coefficients were estimated from Watson et al., ${ }^{1}$ where $D$ was found to have Arrhenius dependence on $T$ for $500{ }^{\circ} \mathrm{C} \leq T \leq 725^{\circ} \mathrm{C}$. Here, $D$ was modeled as having this same dependence for $T \leq 500{ }^{\circ} \mathrm{C}$, using the determined activation energies and pre-exponential factors of $1.37 \mathrm{eV}$ and $1.74 \times 10^{-14} \mathrm{~m}^{2} \mathrm{~s}^{-1}$.

The above $\mathrm{V}_{\mathrm{S}}$ diffusion would lead to formation of an $\mathrm{FeS}_{2-\delta}$ layer on the surface of crystals during reducing anneals. Competing with this, however, is the formation of a layer of pyrrhotite $\mathrm{Fe}_{1-\mathrm{x}} \mathrm{S}$. The thickness of this layer was calculated from values from Lambert et al., ${ }^{2}$ who fit the decomposition rate of pyrite $\left(\mathrm{FeS}_{2}\right)$ to pyrrhotite $\left(\mathrm{Fe}_{1-\mathrm{x}} \mathrm{S}\right)$ with an Arrhenius dependence on $T$, resulting in an activation energy of $2.05 \mathrm{eV}$ and a pre-exponential factor of $1.08 \times 10^{7} \mathrm{~mol} \mathrm{~cm}^{-2}$ $\min ^{-1}$. To estimate the thickness of this pyrrhotite "skin", this rate was converted to an effective $D$ using

$$
J=-D \frac{\Delta c}{\Delta x},
$$

where $J$ is the decomposition rate (in units of $\mathrm{mol} \mathrm{cm}^{-2} \mathrm{~min}^{-1}$ (a flux)) estimated from Lambert et $a l ., \Delta c$ is the difference in concentration of the diffusing species (S) between the pyrite crystal 
( $\left.\sim 0.08 \mathrm{~mol} \mathrm{~cm}^{-3}\right)$ and the pyrrhotite "skin" $\left(\sim 0.02 \mathrm{~mol} \mathrm{~cm}^{-3}\right)$, and $\Delta x$ is an approximate length scale over which this gradient exists. $10 \mu \mathrm{m}$ was used as a $T$-independent estimate, but note that this choice has little effect on the resulting "skin" thickness. Once $D$ was obtained, an estimate of the "skin" thickness was obtained using $l=\sqrt{D t}$, where $l$ is the "skin" thickness and $t$ is time.

As shown in the plot below (for $t=24 \mathrm{hr}$ ), at all temperatures considered, pyrrhotite skin formation greatly outpaces $\mathrm{V}_{\mathrm{S}}$ diffusion. At $430{ }^{\circ} \mathrm{C}$, for example, the surface layer of $\mathrm{FeS}_{2-\delta}$ is predicted to be about 3-4-nm-thick, compared to a surface pyrrhotite layer of thickness $7 \mu \mathrm{m}$.

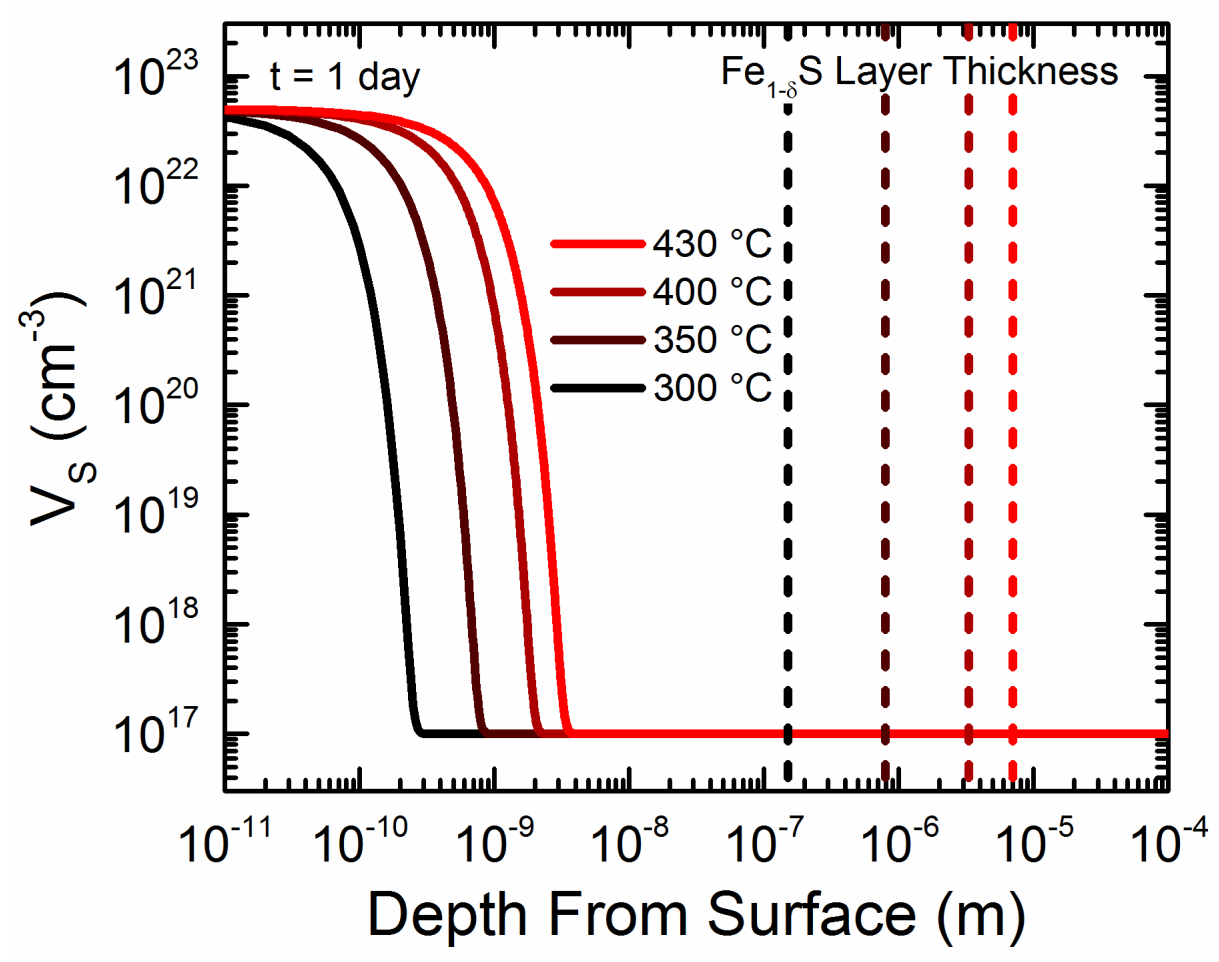

Figure S1. Calculated sulfur vacancy $\left(\mathrm{V}_{\mathrm{S}}\right)$ concentration depth profiles in a pyrite single crystal for a vacuum annealing time of 1 day at different annealing temperatures (solid lines). Annealing temperatures are shown from black (low $T$ ) to red (high $T$ ). Corresponding estimates for the thickness of a $\mathrm{Fe}_{1-\mathrm{x}} \mathrm{S}$ "skin" layer formed by decomposition of the pyrite single crystal under the same conditions are shown with vertical dashed lines. 


\section{B. Representative EDS and PIXE Spectra and Impurity Analysis}

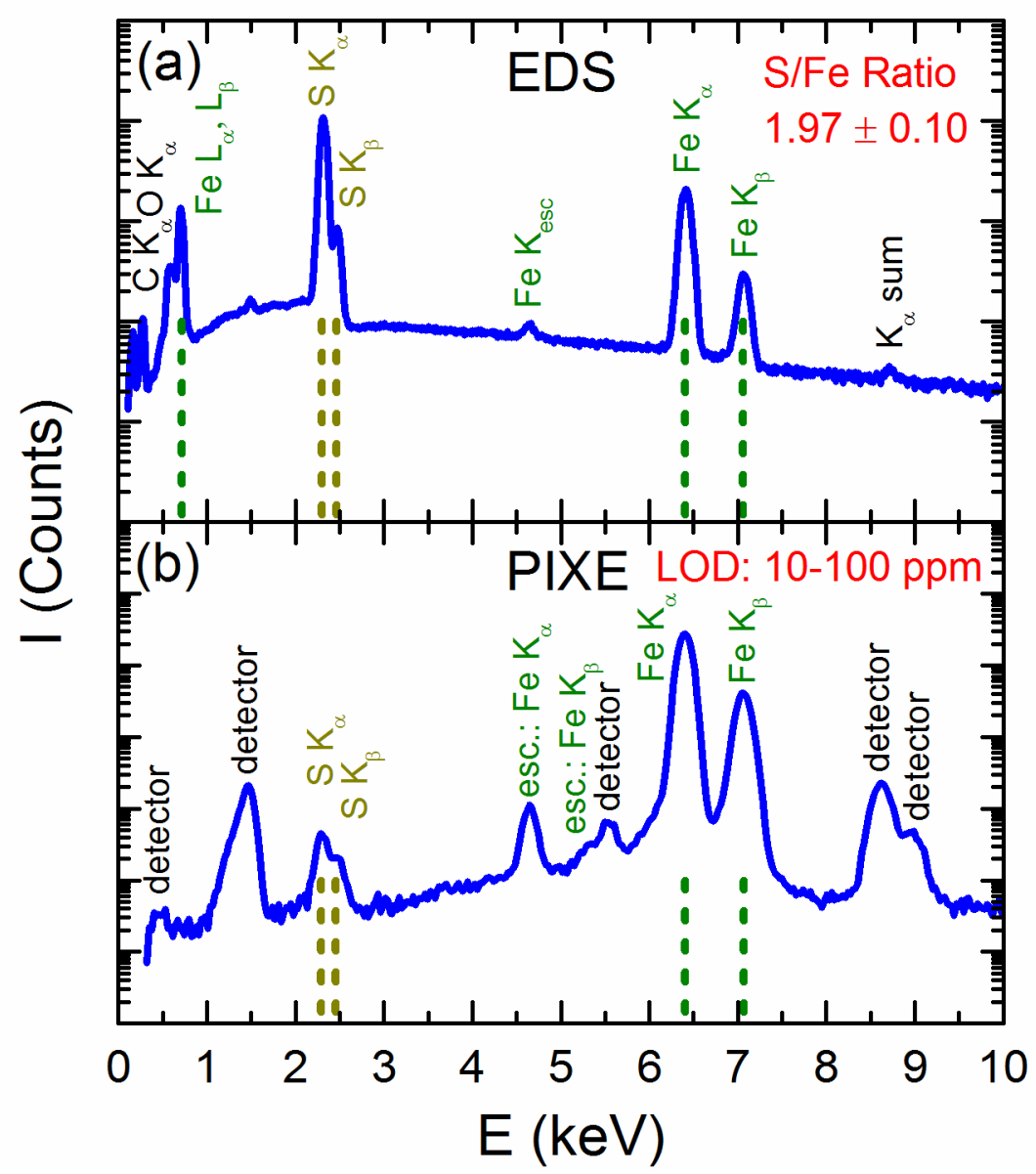

Figure S2. Representative X-ray emission spectra taken on a polished pyrite single crystal grown with standard $\mathrm{FeS}_{2}$ precursor at a $\mathrm{S}: \mathrm{Fe}$ loading of 1.95 with (a) energy-dispersive spectroscopy (EDS) and (b) particle-induced X-ray emission (PIXE). All peaks are labeled in both cases. Also shown is the $\mathrm{S} / \mathrm{Fe}$ ratio calculated from the EDS spectrum. 


\section{Certificate of analysis}

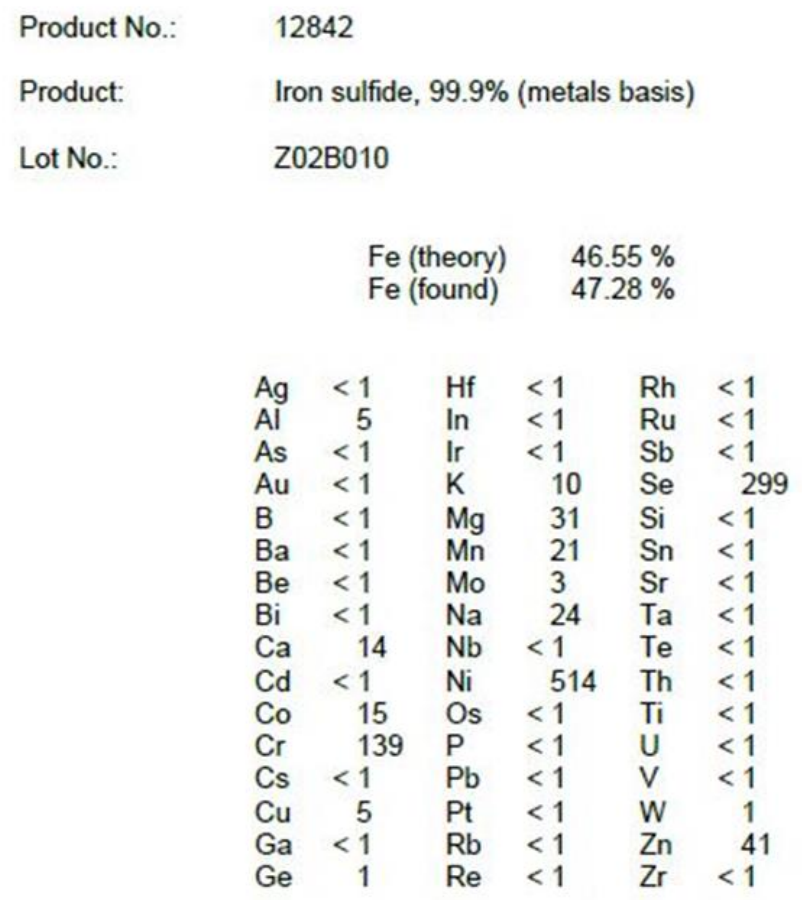

X-Ray Diffraction: Major $\mathrm{FeS}_{2}$ matches PDF 42-1340 Cubic

This document has been electronically generated and does not require a signature.

Order our products online www.alfa.com

Figure S3. Certificate of analysis for the batch of commercial $\mathrm{FeS}_{2}$ precursor powder used in the CVT growth of $\mathrm{FeS}_{2}$ single crystals. 


\section{Summary of Individual Transport Quantities with S:Fe loading}

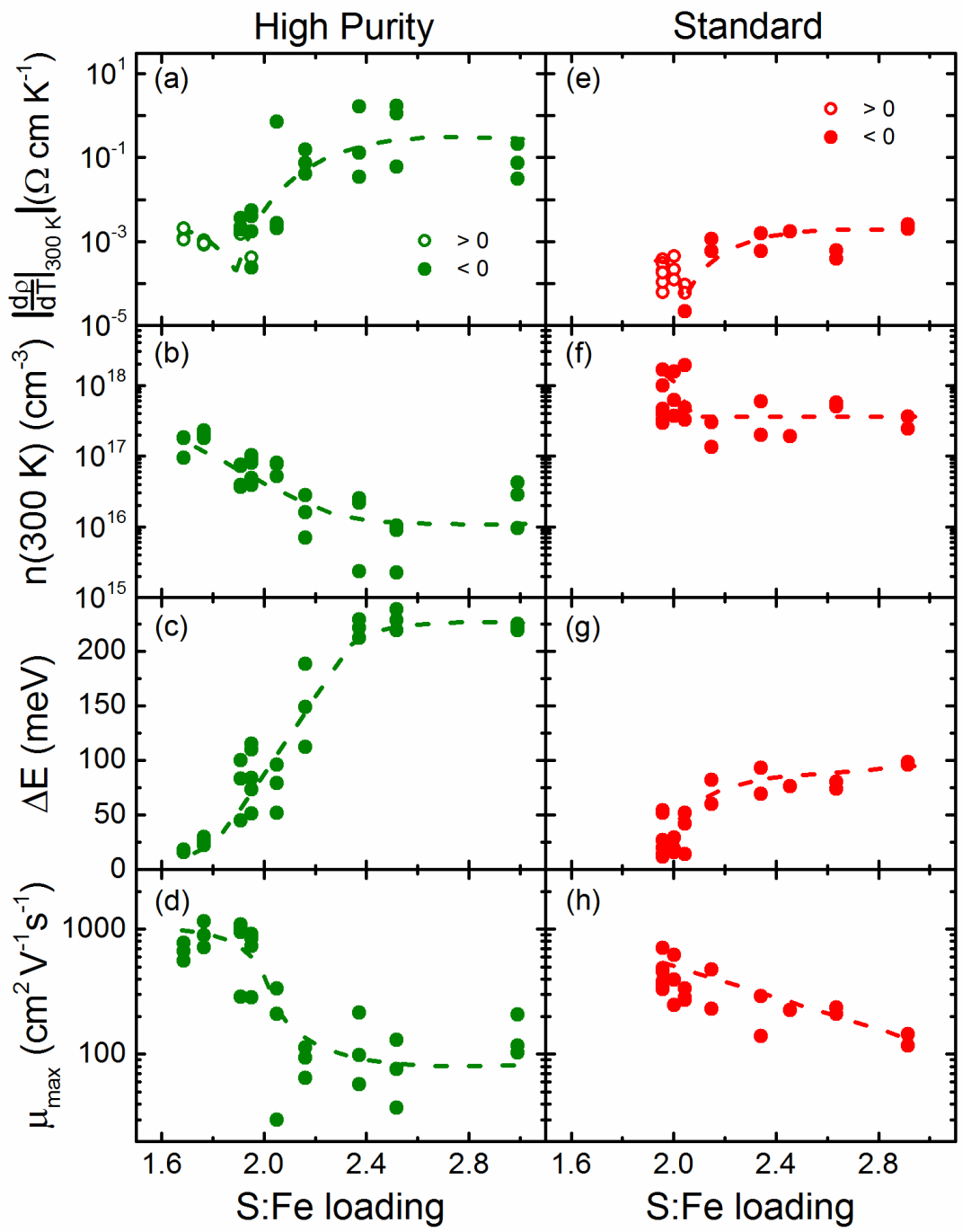

Figure S4. Electronic transport properties of $\mathrm{FeS}_{2}$ crystals vs. S:Fe loading. Crystals grown from (high purity) lab-synthesized precursor powder are shown on the left (panels (a)-(d), green points), with crystals from commercial $\mathrm{FeS}_{2}$ powder (standard) on the right (panels (e)-(h), red points). In all cases the data points are individual crystal transport quantities. Shown are the S:Fe loading dependence of: $(\mathrm{a}, \mathrm{e})$ The $300 \mathrm{~K}$ absolute value of the temperature $(T)$ derivative of resistivity ( $\rho$ ) (open circles denote positive (metallic-like) $d \rho / d T$, closed circles negative (insulating-like) $d \rho / d T)$; (b,f) The $300 \mathrm{~K}$ Hall electron density $(n) ;(\mathrm{c}, \mathrm{g})$ The activation energy $(\Delta E)$ extracted from Arrhenius plots of $n(T)$; $(\mathrm{d}, \mathrm{h})$ The maximum Hall mobility $(\mu)$ in the $175-$ $400 \mathrm{~K} T$ range. Dashed lines are guides to the eye. 
D. FeS 2 Density-of-States Schematics for Crystals Grown with High Purity and Standard Precursor, Based on Electronic Transport Data

High Purity

(a)

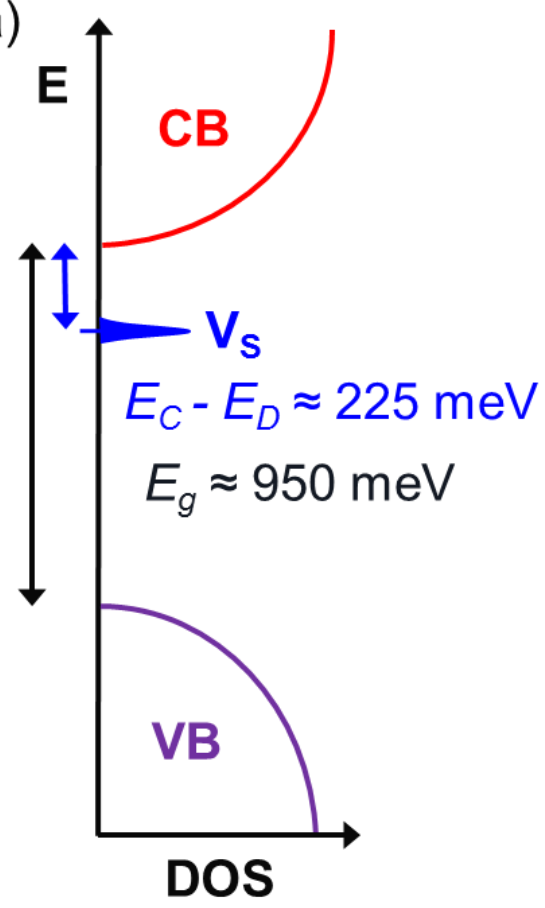

Standard

(b)

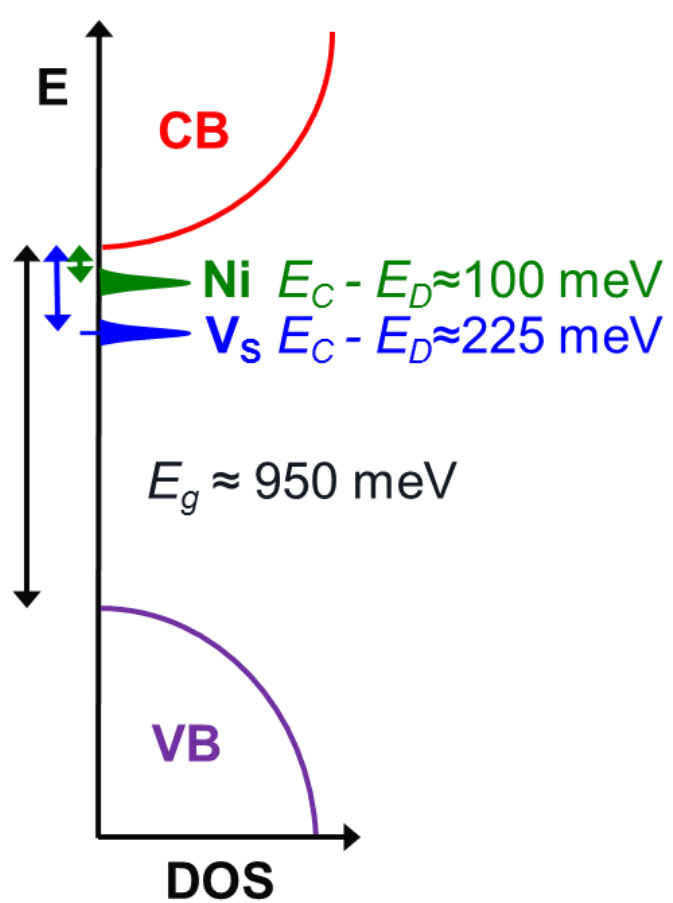

Figure S5. Density-of-states (DOS) schematics for $\mathrm{FeS}_{2}$ crystals grown with (a) high purity, and (b) standard purity precursor powder at high S:Fe loading. Conduction and valence bands are labeled CB and VB. The (mildly broadened for visualization purposes) $\mathrm{V}_{\mathrm{S}}$ and (potential) Ni donor bands are shaded in blue and green, respectively. 


\section{E. Possible Explanations for the Observation of $\Delta E \approx 100 \mathrm{meV}$ in Crystals Grown with Standard Purity Precursor}

Explanation 1: Additional $n$-doping (from MBI) in standard purity crystals shifts the Fermi level during growth, modifying the enthalpy of formation of charged $V_{S}$, and thus the $V_{S}$ concentration at a given $\mathrm{S}$ vapor pressure. This would alter the correspondence between $\mathrm{S}: \mathrm{Fe}$ loading and $\mathrm{V}_{\mathrm{S}}$ concentration, making direct comparisons between Figure 5c,g invalid.

Explanation 2: MBI modify the energy of the $\mathrm{V}_{\mathrm{S}}$ states. Although this would require a $\sim 125$ $\mathrm{meV}$ shift (from $\sim 225$ to $\sim 100 \mathrm{meV}$ ) with MBI concentrations of only $\sim 500 \mathrm{ppm}$, this may be possible through formation of defect complexes, particularly if MBI and $\mathrm{V}_{\mathrm{S}}$ concentrations are of a similar magnitude (see below).

Explanation 3: $\mathrm{Ni}$ is a donor with $\Delta \mathrm{E} \approx 100 \mathrm{meV}$ and is present in large enough concentration to dominate electronic transport

In the following discussion, we investigate in detail the feasibility of explanation 3 . The central question is: If $\mathrm{Ni}$ acts as a donor with $\Delta E \approx 100 \mathrm{meV}$, is it present (in crystals grown with standard precursor powder at high S:Fe loading) in large enough concentration to dominate electronic transport? In other words, would such crystals appear as if they were Ni-dominated (i.e., with $\Delta E \approx 100 \mathrm{meV}$, not the $\Delta E \approx 225 \mathrm{meV}$ expected from $\mathrm{V}_{\mathrm{s}}$ ), at least at high $\mathrm{S}: \mathrm{Fe}$ loading?

The following assumptions were made in calculations:

1. $\mathrm{FeS}_{2}$ crystals are compensated, regardless of whether standard or high purity precursor powder is used. This is discussed in detail in the main text.

2. $\mathrm{V}_{\mathrm{S}}$ are deep donors with $\Delta E=225 \mathrm{meV}\left(\Delta E \equiv E_{C}-E_{D}\right.$, where $E_{C}$ is the energy of the conduction band minimum and $E_{D}$ is the energy of the donor band, due to $\mathrm{V}_{\mathrm{S}}$ in this case)

3. Ni are deep donors with $\Delta E=100 \mathrm{meV}$ and $N_{D, N i} \approx 3 \times 10^{19} \mathrm{~cm}^{-3}(\sim 400 \mathrm{pm}$, as determined from ICPMS)

To describe the transport behavior, one must estimate both $\mathrm{V}_{\mathrm{S}}$ and acceptor concentrations. In a compensated semiconductor, $n(T)$ can be described by 


$$
n(T)=\frac{N_{C}\left(N_{D}-N_{A}\right)}{N_{A}} e^{-\Delta E / k_{B} T}
$$

where $N_{C}$ is the effective density-of-states in the conduction band, $N_{D}$ is the donor concentration, $N_{A}$ is the acceptor concentration, $k_{B}$ is Boltzmann's constant, and $T$ is temperature. ${ }^{3}$ To estimate the $\mathrm{V}_{\mathrm{S}}$ donor concentration $\left(N_{D, V S}\right)$, which we assume is independent of which precursor was used, we apply

$$
N_{C}(300 K)=2\left(\frac{2 \pi m_{e}{ }^{*} k_{B} T}{h^{2}}\right)^{3 / 2}=7.6 \times 10^{18} \mathrm{~cm}^{-3}
$$

where $m_{e}{ }^{*}$ is the effective electron mass $\left(0.45 m_{e}\right)^{4}$ and $h$ is Planck's constant. We estimate $N_{A} \approx$ $7.5 \times 10^{17} \mathrm{~cm}^{-3}(10 \mathrm{ppm})$, from the concentration of MBI in crystals grown with high purity precursor. This implies one or more MBIs are acceptors; it is possible, even likely, that Fe vacancies or other defects could be acceptors, and present in concentrations at or above $10 \mathrm{ppm}$, but we have no evidence of this. Using these $N_{A}$ and $N_{C}$ values, we find $N_{D, V s} \approx 9.3 \times 10^{18} \mathrm{~cm}^{-3}$.

The temperature dependence of the Fermi energy $\left(E_{F}\right)$ and electron density $(n)$ was then calculated using non-degenerate carrier statistics, solving the charge neutrality condition $(p+$ $N_{D}{ }^{+}-n-N_{A}{ }^{-}=0$, where $p$ is the hole density, $N_{D}{ }^{+}$is the ionized donor concentration, and $N_{A}^{-}$is the ionized acceptor concentration) for temperatures $<400 \mathrm{~K}$. To understand how $E_{F}(T)$ and $n(T)$ evolve with the inclusion of dopants, we start with the simplest case: uncompensated $\left(N_{A}=0\right) \mathrm{V}_{\mathrm{S}}$-doped $\mathrm{FeS}_{2}$ (with no Ni), with $N_{D, V s}=9.3 \times 10^{18} \mathrm{~cm}^{-3}$ and $\Delta E=225 \mathrm{meV}$ (dotted green lines in Figures S6a,b). We then introduce $N_{A}=7.5 \times 10^{17} \mathrm{~cm}^{-3}$ (solid green lines in Figures S6a-c). Compensation is clearly evident with inclusion of $N_{A}, E_{F}$ and $n$ notably decreasing at all $T$ relative to their values when no acceptors are present. When plotting $n(T)$ in Arrhenius fashion to extract $\Delta E, 255 \mathrm{meV}$ is obtained, in agreement with the $225 \mathrm{meV}$ input into the calculation, albeit slightly increased due to the additional temperature dependence of $N_{C}$. Next, we modeled uncompensated $\left(N_{A}=0\right) \mathrm{FeS}_{2}$ with both $\mathrm{V}_{\mathrm{S}}$ (with the $\Delta E$ and $N_{D, V s}$ from above) and $\mathrm{Ni}$ (with $\Delta E$ and $N_{D, V s}$ as outlined in assumption 3). $E_{F}(T)$ and $n(T)$ are shown as the dotted red line in Figures S6a,b. We then introduced $N_{A}=7.5 \times 10^{17} \mathrm{~cm}^{-3}$ (solid red line in Figures S6a-c). Here, compensation is still evident below $\sim 300 \mathrm{~K}$. This is consistent with the universal $\mu(n)$ relation described in the main text. Critically, inclusion of $\mathrm{Ni}$ significantly 
increases $E_{F}$ and $n$ at all $T$, and fitting $n(T)$ in Arrhenius fashion yields $\Delta E=93 \mathrm{meV}$, in good agreement with the $\Delta E$ of $\mathrm{Ni}$ included in the calculation. This suggests that $\mathrm{Ni}$, at $\sim 400 \mathrm{ppm}$ in crystals grown with standard precursor, is present in large enough concentration to overwhelm the impact of $\mathrm{V}_{\mathrm{S}}$ and lower $\Delta E$ to near $100 \mathrm{meV}$, if it acts as a donor with this $\Delta E$. Note that this is not clear evidence that $\mathrm{Ni}$ is a donor in $\mathrm{FeS}_{2}$ with $\Delta \mathrm{E}=100 \mathrm{meV}$, but rather an indication that we cannot rule it out as an explanation for a change in $\Delta \mathrm{E}$ upon the inclusion of $\mathrm{Ni}$.

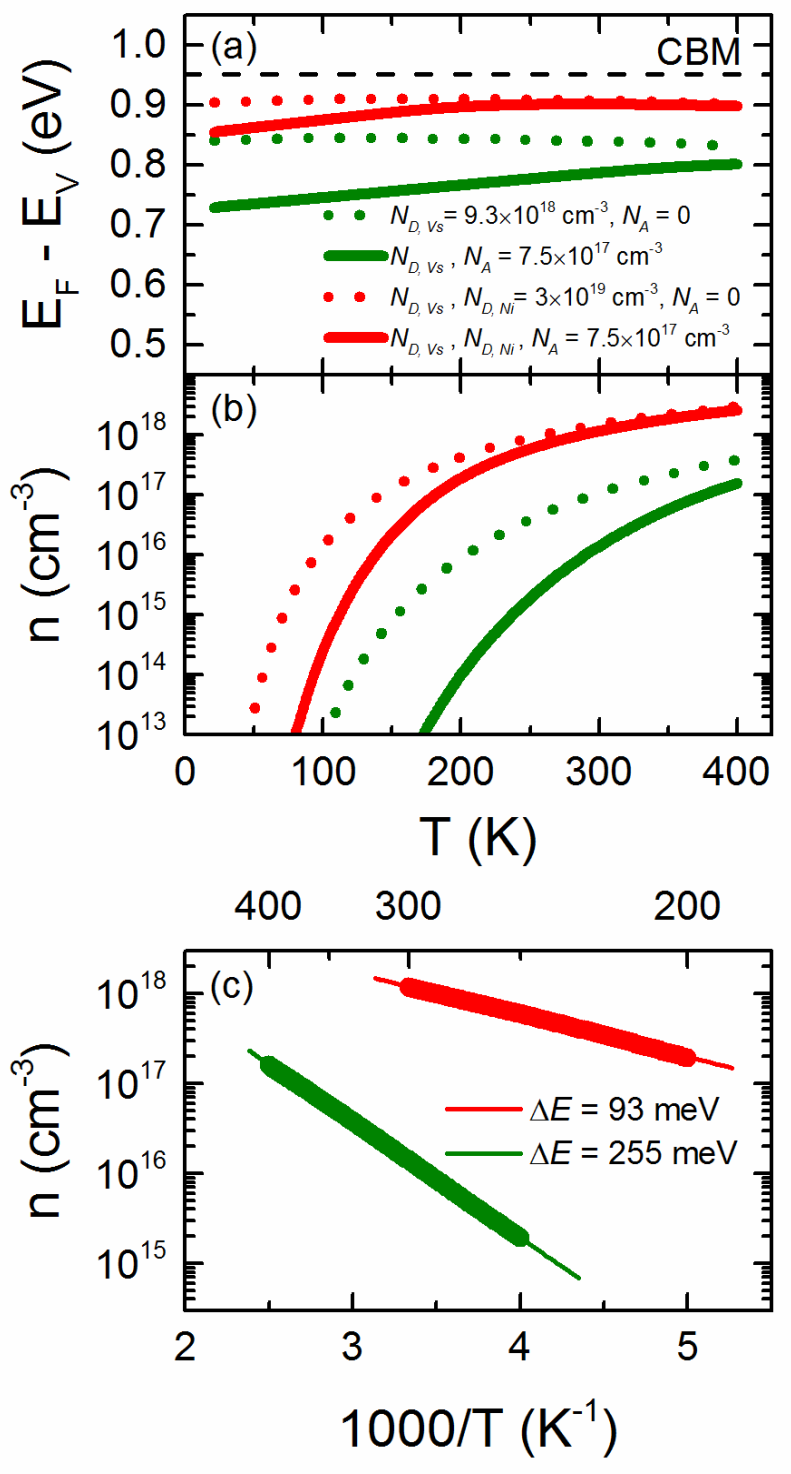

Figure S6. Temperature dependence of the (a) Fermi energy $\left(E_{F}\right)$ and (b) electron density $(n)$, calculated with different dopants $\left(\mathrm{V}_{\mathrm{S}}, \mathrm{Ni}\right.$, and an unidentified acceptor $\left.N_{A}\right)$. (c) Arrhenius analysis of the $n(T)$ shown in (b), showing that the resulting $\Delta E$ values reflect the $\mathrm{V}_{\mathrm{S}}$ (green) and $\mathrm{Ni}$ (red) donor band positions relative to the conduction band minimum (CBM). 


\section{References}

(1) Watson, E. B.; Cherniak, D. J.; Frank, E. A. Retention of Biosignatures and MassIndependent Fractionations in Pyrite: Self-Diffusion of Sulfur. Geochim. Cosmochim. Acta 2009, 73, 4792-4802.

(2) Lambert, J. M., Jr.; Simkovich, G.; Walker, P. L., Jr. The Kinetics and Mechanism of the Pyrite-to-Pyrrhotite Transformation. Metall. Mater. Trans. B 1998, 29B, 385-396.

(3) Ashcroft, N. W.; Mermin, N. D. Solid State Physics; Harcourt College, 1976.

(4) Karguppikar, A. M.; Vedeshwar, A. G. Transport Properties of Thin Iron Pyrite Films. Phys. Status Solidi 1986, 95, 717-720. 